\title{
An Efficient Two step Algorithm for Despeckling the Ultrasound Image
}

\author{
Nageswari P, Rajan S, Manivel K
}

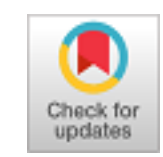

\begin{abstract}
The speckle noise presence in ultrasound images is a critical concern in medical image processing. It degrades the important features captured in an image and decreases the physician's capacity to understand the image accurately. In recent years, numerous techniques have been proposed to de-noise the ultrasound images. In this paper, a new speckle noise removal algorithm has been proposed for medical ultrasound images. Based on the concepts of fuzzy logic and Coefficient of variation, the proposed algorithm first classifies the image area into three different regions such as homogeneous, edge and detail region. Next, average filter, median filter and an adaptive mean filter are employed to partition the unwanted noise from the pixels of different regions. Filter selection depends on the features of a region. The proposed algorithm develops image quality by removing maximum unwanted noise while protecting the important image details.
\end{abstract}

Index Terms: Ultrasound Image, Fuzzy Logic, Triangular Membership function, SpeckleNoise, Image Fuzzification

\section{INTRODUCTION}

The process of medical imaging is to capture the images of internal organs and soft structural tissues of the human body. Currently, different imaging modalities are available in medical field. Out of these modalities, ultrasound imaging is often considered because of its versatile, portable, non-invasive, real time imaging and relatively cost effective. On the other hand, the main drawback of medical ultrasound imaging is that, it intrinsically degrades with speckle noise. This drawback creates an opportunity for a physician to take a wrong decision about an image with speckle noise. Hence denoising has become an essential preprocessing task in medical image processing. Speckle noise that affects ultrasound images is a multiplicative noise which follows gamma distribution.

\section{RELATED WORKS}

Filters [1, 2] based on local statistics provide efficient denoising in homogeneous region however fail to protect the

Revised Manuscript Received on October 30, 2019.

* Correspondence Author

Nageswari P*, Research Scholar, Department of Mathematics, Erode Arts and Science College, Erode, Tamilnadu, India.

Dr.Rajan S, Department of Mathematics, Erode Arts and Science College, Erode, Tamilnadu, India.

Dr.Manivel K, Department of ECE, Mahendra Engineering College, Namakkal, Tamilnadu, India.

(C) The Authors. Published by Blue Eyes Intelligence Engineering and Sciences Publication (BEIESP). This is an open access article under the CC BY-NC-ND license (http://creativecommons.org/licenses/by-nc-nd/4.0/) noisy pixels in edge region. Yonjian et al. [3] and Krissian et al.[4] proposed diffusion algorithms for speckle reducing in ultrasound images. This algorithm modifies the image through partial differential equation. Rudin et al. [5] introduced a new technique which replaced the multiplicative noise model into additive Gaussian noise. This technique executes well only for additive noise model. Coll et al. [6] presented a non- local pattern for ultrasound images based on patches. This pattern is mainly introduced for de-noising the image with white Gaussian noise and it produced good results when compared with other art filters. Farzana et al. [7] proposed a de-speckling algorithm based on adaptive bilateral filtering. Of late, many researchers focus their interest to involve fuzzy techniques in medical image denoising process. It has the capability to handle the image with vagueness and ambiguity efficiently. Also it represents and manages the human facts as fuzzy if-then rules. Filters based on fuzzy logical system provide a good solution for classical logical system based filters [8]. Based on fuzzy techniques, Cheng et al. [9] found a new speckle noise reducing method, for synthetic radar images. In this method, for every pixel in the filtering window, fuzzy edges are calculated and fuzzy filtering is done by employing the weight contributions of adjacent pixels. However the main drawback of this method is that, it is appropriate only for homogeneous regions. Zhang et al. [10] presented a fuzzy logic and sub pixel fractional diffusion approach for de-speckling the ultrasound images. This approach employs Euler Lagrange equation and does the filtering in an iterative way which improves the image contrast. But there is a limitation for calculating the image Fuzzification for every iteration Binaee et al. [11] proposed an ultrasound image enhancement methodology by utilizing gradient for image classification and calculating non local mean for similar windows. Babu et al. [12] discussed an adaptive speckle reducing technique for ultrasound images. Initially, based on coefficient of variation, this technique classifies the image area into three different regions. Then the filtering is carried out by selecting appropriate filters.Based on discrete topological derivative, an ultrasound de-speckling algorithm is proposed by Damodaran et al. [13].This algorithm reduces speckle noise proficiently but it consumes more time to improve the image contrast. 


\section{An Efficient Two step Algorithm for Despeckling the Ultrasound Image}

Similarly, based on multi transducer architecture, a de-noising method is developed by Tsakalakis et al. [14]. This method employed a technique which combines spatial and frequency compounding with super solution de-speckling algorithm. The major disadvantage is, the image registration is required for the images which are captured from various sensors with dissimilar frequencies.

Based on the concepts of fuzzy logic and Coefficient of variation, the proposed algorithm, first classifies the image area into three different regions such as homogeneous, edge and detail region. Next, Average Filter, Median Filter and an Adaptive Mean Filter are employed to breakdown the obnoxious noise from the pixels of different regions.

The paper is organized as follows: Section 2 describes the related work, their limitations and the objective of the paper. Section 3 discusses the proposed method; section 4 and 5 describes the simulation results and conclusion.

\section{PROPOSED WORK}

In the proposed method, the image areas are divided into three different regions and fuzzy membership function is employed to sketch the outer line among these regions.

Further the image is classified by using the coefficient of variation. Coefficient of variation is defined as the ratio of standard deviation and mean. A pixel with minimum value of coefficient of variation determines a homogeneous region pixel, maximum value of coefficient of variation denotes edge pixels and intermediate value corresponds to the detail region. Depending on this principle, the noisy pixels have been classified into three distinct regions namely homogeneous, edge and detail regions. Then a fuzzy triangular membership function has been employed to address the speckle noise fuzziness. It is utilized to describe fuzzy values of different image regions.

Let us consider the ultrasound image that degrades with speckle noise be $I(y, z)$.

Then

$$
I(y, z)=N(y, z) \cdot M(y, z)
$$

Where, $N(y, z)$ is the noise free image and $M(y, z)$ is the multiplicative speckle noise. $P \times Q$ is the dimensions of the image $I(y, z)$. Where $y=1 \ldots P$ and $z=1 \ldots Q$.

The following equation defines the fuzzy membership value for each pixel of the image $I(y, z)$.

$$
I(y, z)= \begin{cases}\mu_{\text {Homo }}=\frac{n-C O V}{n-l} & l \leq C O V \leq p_{1}, \\ \mu_{\text {Detail }}=\max \left(\min \left(\frac{C O V-p_{1}}{n-p_{1}}, \frac{p_{1}-C O V}{p_{2}-n}\right), 0\right) & p_{1} \leq C O V \leq p_{2} \\ \mu_{\text {Edge }}=\frac{C O V-n}{m-n} & \text { otherwise }\end{cases}
$$

Where, $y=1 \ldots \ldots . P, z=1 \ldots \ldots Q$

Based on the principle of Coefficient of variation, the image pixels are separated into three different regions namely, homogeneous detail and edge region. Pixel belonging to the homogeneous region has low $\mathrm{COV}$ whereas high $C O V$ shows edge region and medium belonging to the detail region.

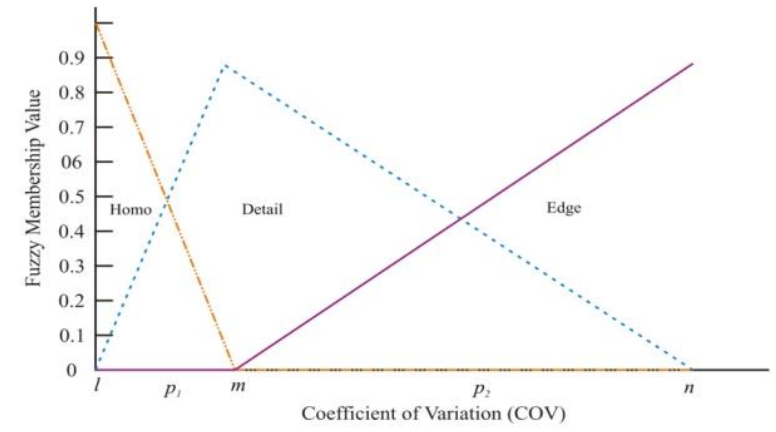

Fig. 1 Triangular Memership Function

Figure 1 shows the fuzzy membership function for the image $I(y, z)$.

The details of calculating threshold values are given below

$$
\begin{aligned}
& l=\min \left(C O V_{k}\right) \\
& m=\max \left(\operatorname{COV}_{k}\right) \\
& n=\operatorname{middle}\left(\text { Unique }_{\left(C O V_{k}\right)}\right) \\
& p_{1}=\text { average }\left(\operatorname{COV}_{[l, n]}\right) \\
& p_{2}=\text { average }\left(\operatorname{COV}_{[n, m]}\right)
\end{aligned}
$$

Where, $k \epsilon P x Q$, single $\left(C O V_{k}\right)$ represents, if its applied to a vector, then the outcome would include no monotonous value. $[l, n],[n, m]$ denote the range. $\operatorname{COV}_{[l, n]}$ represents all value of $C O V$ among thresholds $l$ and $n$. Similarly $C O V_{[n, m]}$ represents all values of $C O V$ among thresholds $n$ and $m$. Figure 2 shows the flow diagram of the proposed method.

\section{A. Algorithm:}

1. Compute coefficient of variation employing 3x3 window in

the region of each pixel of $I(y, z)$

2. Compute triangular membership function thresholds by using

$\mathrm{l}=\min \left(C O V_{\mathrm{k}}\right)$

$\mathrm{m}=\max \left(C O V_{\mathrm{k}}\right)$

$\mathrm{n}=$ middle (Unique $\left.\left(C O V_{\mathrm{k}}\right)\right)$

$\mathrm{p}_{1}=$ average $\left(C O V_{[1, \mathrm{n}]}\right)$

$\mathrm{p}_{2}=$ average $\left(C O V_{[n, m]}\right)$

Where, $k \in P x Q$

3. Isolate every pixel of image I into distinct region employing triangular function

$$
I(y, z)= \begin{cases}\mu_{\text {ното }}=\frac{n-\mathrm{COV}}{n-l} & l \leq \mathrm{COV} \leq p_{1}, \\ \mu_{\text {Detail }}=\max \left(\min \left(\frac{\mathrm{COV}-p_{1}}{n-p_{1}}, \frac{p_{1}-\mathrm{COV}}{p_{2}-n}\right), 0\right) & p_{1} \leq \mathrm{COV} \leq p_{2} \\ \mu_{\text {Edge }}=\frac{\operatorname{COV}-n}{m-n} & \text { otherwise }\end{cases}
$$

4. Employ suitable filter to denote each pixel of all three regions

$$
D^{\prime}(y, z)= \begin{cases}\vartheta_{1} & I(y, z) \in \mu_{\text {Ното }} \\ \vartheta_{2} & I(y, z) \in \mu_{\text {Detail }} \\ \vartheta_{3} & I(y, z) \in \mu_{\text {Edge }}\end{cases}
$$

Where, 


$$
\begin{gathered}
\vartheta_{1}=\frac{1}{q} \sum_{j=-1}^{1} \sum_{k=-1}^{1} I(y+j, z+k) \\
\vartheta_{2}=\operatorname{median}( \pm(y+j, z+k)) \text { Where, }-1 \leq(j, k) \leq 1 \\
\vartheta_{3}=\frac{\sum_{j=-1}^{1} \sum_{k=-1}^{1} W(i, j) \times I(y+j, z+k)}{\sum_{j=-1}^{1} \sum_{k=-1}^{1} W(i, j)}
\end{gathered}
$$

5. Obtain the De-noised Image $D^{\prime}(y, z)$.

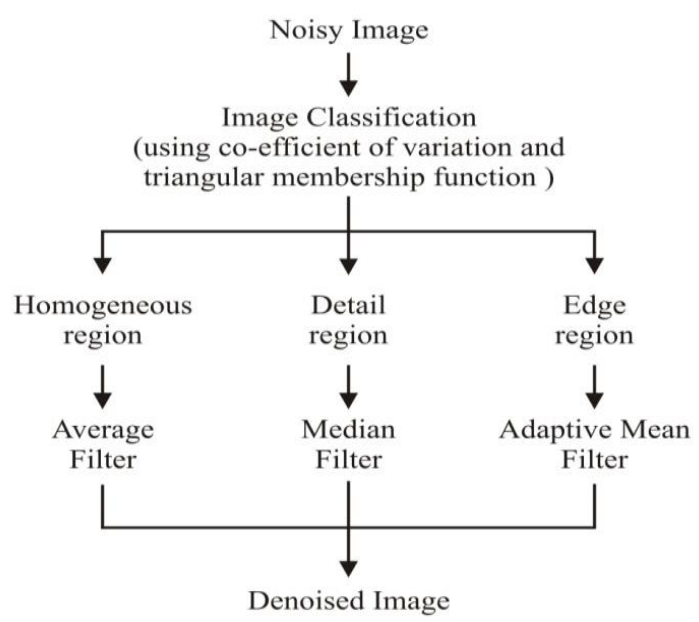

Fig. 2 Flow diagram of the proposed method

\section{B. Adaptive Speckle De-Noising:}

To obtain a de-noised image, an appropriate filter will be employed on every pixel of the image $H$ which is classified into homogeneous, edge and detail region pixels. Generally, the noise effect in homogeneous region is very low when compared to edge or detail region. Hence an average filter is enough to remove noise in homogeneous region. For detail region we need to preserve the structural details. Therefore, a structural preserving non-linear filter, median filter would be used for filtering the detail region. In edge region, there is a chance to have both affected and non affected edges. Thus, an efficient filter would be required for denoising the noisy edge pixels to protect edges without distressing the original image content.

\section{(i)Average Filter for Homogeneous Region:}

$\mathrm{D}^{\prime}(\mathrm{y}, \mathrm{z})$, the new restored pixel value after processing the homogeneous region pixels with average filter, is given by

$$
D^{\prime}(y, z)=\frac{1}{9} \sum_{j=-1}^{1} \sum_{k=-1}^{1} I(y+j, z+k)
$$

\section{(ii) MEDIAN FILTER FOR DETAIL REgION:}

Median filter has the capacity to eliminate noise while protecting detail regions. Hence, we have utilized this filter to deal with detail region.

The equation for median filter with moving window of size $3 \times 3$ is given as follows:

$$
D^{\prime}(y, z)=\operatorname{Med}(I(y+j, z+k))
$$

Where, $-1 \leq(j, k) \leq 1$

(iii) Adaptive Mean Filter:
Edge pixels are continuous and grouped in nature. Hence we have to assign larger weight to the co-efficient of neighboring pixel with the same magnitude. Alternatively, noisy pixels which have larger co-efficient are discontinuous and isolated in nature. On the basis of this study, an adaptive weighted mean filter is used for filtering the edge noisy pixels. Therefore we have to assign smaller weight to the co-efficient of neighboring pixel with different magnitude. For every neighboring pixel with different magnitude for every neighboring co-efficient, the weight value is given by

$$
D^{\prime}(y, z)=\frac{\sum_{j=-1}^{1} \sum_{k=-1}^{1} w(j, k) \times I(y+j, z+k)}{\sum_{j=-1}^{1} \sum_{k=-1}^{1} w(j, k)}
$$

$w(j, k)=r(j, k) \times s(j, k)$

Where, $s(j, k)$ and $r(j, k)$ are the spatial and magnitude similarity respectively denoted as in [15].

The magnitude and spatial similarity is given by

$$
\begin{gathered}
r(j, k)=e^{\left[-\left(\frac{I(y, z)-I(y+j, z+k)}{S}\right)^{2}\right]} \\
s(j, k)=e^{\left[-\left(\frac{j^{2}+k^{2}}{9}\right)\right]}
\end{gathered}
$$

Where $I(y, z)$ and $I(y+i, z+k)$ are middle pixel and its adjacent pixel respectively in a $3 \times 3$ window and $j, k \in[-1$ to 1$]$.

\section{SIMULATION RESULTS:}

The proposed speckle noise reducing method is implemented in MATLAB 14a and simulation results are presented. The performance is compared with Mean, Median, AMF, AWMF, LEE, ASSF, ANSF, ABF and Fuzzy Filter. For quantitative analysis, performance of different filters is compared in terms of Mean Square Error (MSE) and Peak Signal to Noise Ratio (PSNR).

Mean Square Error (MSE) is defined as:

$$
M S E=\frac{\sum_{i j}\left(r_{i j}-X_{i j}\right)^{2}}{M N}
$$

Peak Signal to Noise Ratio (PSNR) is defined as:

$$
\mathrm{PSNR}=P S N R=10 \log _{10}\left[\frac{255^{2}}{M S E}\right]
$$

Where, MSE is the mean square error between original image and filtered image.

To test effectiveness of the proposed methodology, real human liver ultrasound image has been considered. Figure 3 shows the residual images which are obtained by applying various denoising techniques on real liver image. It is obvious from these figures that mean and median filters are able to suppress considerable amount of noise but fails to protect the edge regions. 


\section{An Efficient Two step Algorithm for Despeckling the Ultrasound Image}

AMF and AWMF creates lots of vagueness. During the process of denoising, filters introduced by M. Karama et.al and D. Kuan et.al removes the important structural details. Fuzzy filter produces no significant performance in terms of both edge preservation and noise suppression.
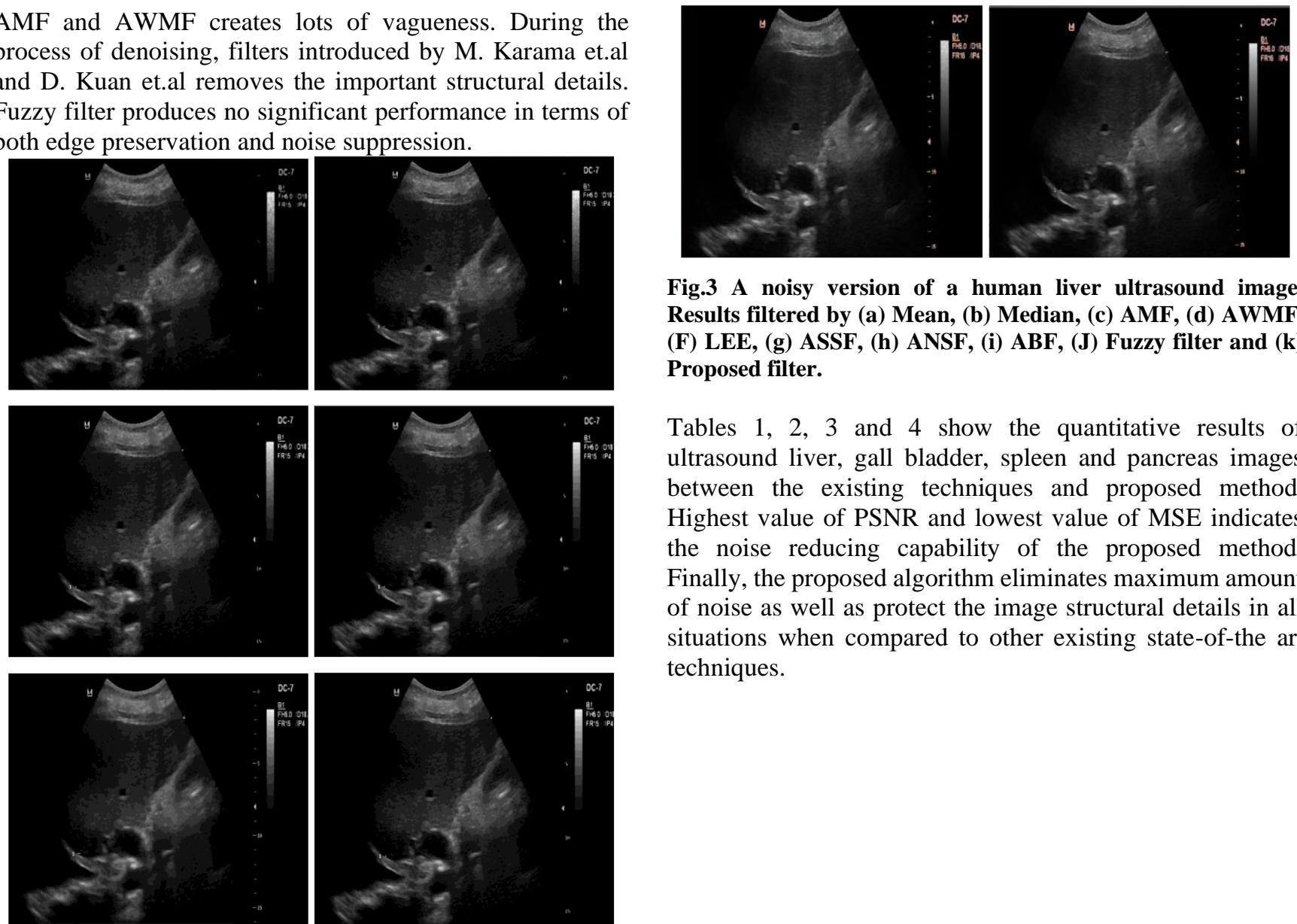

Fig.3 A noisy version of a human liver ultrasound image. Results filtered by (a) Mean, (b) Median, (c) AMF, (d) AWMF, (F) LEE, (g) ASSF, (h) ANSF, (i) ABF, (J) Fuzzy filter and (k) Proposed filter.

Tables 1, 2, 3 and 4 show the quantitative results of ultrasound liver, gall bladder, spleen and pancreas images between the existing techniques and proposed method. Highest value of PSNR and lowest value of MSE indicates the noise reducing capability of the proposed method. Finally, the proposed algorithm eliminates maximum amount of noise as well as protect the image structural details in all situations when compared to other existing state-of-the art techniques.

Table: 1 Quantitative analysis of ultrasound liver image:

\begin{tabular}{|c|c|c|c|c|c|c|c|c|}
\hline \multirow{2}{*}{$\begin{array}{c}\text { Nois } \\
\text { algorithm }\end{array}$} & \multicolumn{2}{|c|}{ Speckle noise (10\%) } & \multicolumn{2}{c|}{$\begin{array}{c}\text { Speckle noise } \\
\text { (20\%) }\end{array}$} & \multicolumn{2}{c|}{ Speckle noise (30\%) } & \multicolumn{2}{c|}{$\begin{array}{c}\text { Speckle noise } \\
\text { (40\%) }\end{array}$} \\
\cline { 2 - 9 } & MSE & PSNR & MSE & PSNR & MSE & PSNR & MSE & PSNR \\
\hline Average & 128.563 & 21.574 & 134.324 & 20.743 & 138.528 & 19.584 & 145.782 & 17.362 \\
\hline Median & 127.533 & 26.948 & 129.335 & 23.948 & 136.243 & 22.683 & 136.983 & 19.566 \\
\hline AMF & 115.684 & 27.547 & 121.854 & 24.547 & 128.225 & 25.852 & 132.582 & 21.254 \\
\hline AWMF & 112.805 & 28.365 & 118.520 & 26.365 & 122.569 & 26.523 & 127.832 & 23.226 \\
\hline Lee & 110.164 & 29.814 & 116.634 & 27.891 & 119.234 & 27.412 & 123.748 & 24.263 \\
\hline ASSF & 108.361 & 31.425 & 112.148 & 29.425 & 115.674 & 29.122 & 121.962 & 27.856 \\
\hline ANSF & 102.482 & 33.432 & 106.872 & 31.432 & 112.336 & 31.326 & 119.783 & 29.845 \\
\hline ABF & 98.564 & 34.896 & 101.634 & 33.896 & 108.568 & 32.258 & 115.543 & 31.482 \\
\hline Fuzzy filter & 94.854 & 37.854 & 98.425 & 34.854 & 107.213 & 34.245 & 113.869 & 32.268 \\
\hline Proposed filter & 87.238 & 38.532 & 92.387 & 35.532 & 105.873 & 35.842 & 111.852 & 34.665 \\
\hline
\end{tabular}

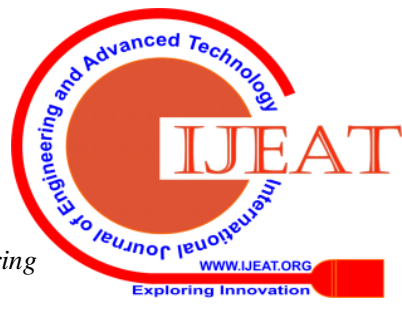


Table: 2 Quantitative analysis of ultrasound gall bladder image:

\begin{tabular}{|c|c|c|c|c|c|c|c|c|}
\hline \multirow{2}{*}{$\begin{array}{c}\text { Noise } \\
\text { algorithm }\end{array}$} & \multicolumn{2}{|c|}{$\begin{array}{c}\text { Speckle noise } \\
(\mathbf{1 0})\end{array}$} & \multicolumn{2}{c|}{$\begin{array}{c}\text { Speckle noise } \\
(\mathbf{2 0} \%)\end{array}$} & \multicolumn{2}{c|}{$\begin{array}{c}\text { Speckle noise } \\
(30 \%)\end{array}$} & \multicolumn{2}{c|}{$\begin{array}{c}\text { Speckle noise } \\
\text { (40\%) }\end{array}$} \\
\cline { 2 - 9 } & MSE & PSNR & MSE & PSNR & MSE & PSNR & MSE & PSNR \\
\hline Average & 129.352 & 22.325 & 135.244 & 21.223 & 141.528 & 19.364 & 148.963 & 18.922 \\
\hline Median & 128.224 & 25.369 & 131.288 & 24.975 & 138.243 & 22.258 & 142.862 & 21.655 \\
\hline AMF & 114.356 & 28.854 & 127.336 & 27.226 & 132.456 & 25.425 & 138.965 & 23.346 \\
\hline AWMF & 113.244 & 30.253 & 120.852 & 29.572 & 126.864 & 26.841 & 133.569 & 24.822 \\
\hline Lee & 111.521 & 32.251 & 118.344 & 28.698 & 121.856 & 27.235 & 126.853 & 25.544 \\
\hline ASSF & 109.142 & 34.672 & 116.254 & 31.258 & 119.364 & 29.954 & 122.582 & 27.622 \\
\hline ANSF & 105.436 & 36.549 & 112.522 & 35.422 & 118.668 & 33.437 & 120.834 & 31.411 \\
\hline ABF & 102.452 & 37.292 & 108.532 & 36.691 & 112.569 & 34.534 & 116.425 & 32.558 \\
\hline Fuzzy filter & 98.546 & 38.854 & 103.854 & 37.522 & 105.336 & 36.246 & 112.544 & 33.452 \\
\hline roposed filter & 91.543 & 39.235 & 96.528 & 38.532 & 98.736 & 37.836 & 110.256 & 34.823 \\
\hline
\end{tabular}

\section{CONCLUSION}

A new speckle denoising methodology for real abdomen images has been given in this paper. The proposed filter is based on the concept of fuzzy logic and coefficient of variation. Filtering process has been carried out in two steps, in step-1image region has been classified into three different regions and in step-2 appropriate filters have been employed. Both quantitatively and qualitatively results have been proved. Hence, we finalize that proposed algorithm can proficiently denoising the abdomen ultrasound image and it too develops diagnostic outcomes of modality of ultrasound image.

\section{REFERENCES}

1. M. Karamra, M.A. Kutay, G. Bozdagi, An adaptive speckle suppression filter for medical ultrasonic imaging, IEEE Trans. Med. Imaging 14 (1995) 283-292.

2. D. Kuan, A. Sawchuck, T. Strand, P. Chavel, Adaptive noise smoothing filter for images with signal dependent noise, IEEE Trans. Pattern Anal. Mach. In tell. 7(2) (1985) 165-177.

3. Y. Yongjian, S.T. Acton, Speckle reducing anisotropic diffusion, IEEE Trans. Image Process. 11 (11) (2002) 1260-1270.

4. K. Krissian, C.F. Westin, R. Kikinis, K.G. Vosburgh, Oriented speckle reducing anisotropic diffusion, IEEE Trans. Image Process. 16 (5) (2007) 1412-1424.

5. L.I. Rudin, et al., Nonlinear total variation based noise removal algorithms, Physica D 60 (1992) 259-268.

6. B. Coll. Buades, J.M. Morel, Nonlocal image and movie denoising, Int. J. Comput. Vis. 76 (2) (2008) 123-139.

7. E. Farzana, M. Tanzid, K.M. Mohsin et al., in Proceedings of IEEE-TENCON. Adaptive bilateral filtering for despeckling of medical ultrasound images (Japan, 2010), pp. 1728-1733. doi:10.1109/tencon.2010.5686140.

8. Yager, Ronald R., and Lotfi A. Zadeh, 1992, ‘An Introduction to Fuzzy Logic Applications in Intelligent Systems', Kluwer Academic Publishers Norwell, MA, USA.

9. H. Cheng, J. Tian, Speckle reduction of synthetic aperture radar images based on fuzzy logic, in: First International Workshop on Education Technology and Computer Science, IEEE Computer Society, Wuhan, Hubei, 2009, pp. 933-937.

10. Y. Zhang, H.D. Cheng, J. Tian, J. Huang, X. Tang, Fractional sub pixel diffusion and fuzzy logic approach for ultrasound speckle reduction, Pattern Recognition. 43 (8) (2010) 2962-2970.

11. K. Binaee, R.P.R. Hasanzadeh, An ultrasound image enhancement method using local gradient based fuzzy similarity. Biomed. Signal Process. Control 13, 89-101 (2014). doi:10.1016/j.bspc.2014.03.013

12. J. Babu, J. Jaganath, G.F. Sudha, Adaptive speckle reduction in ultrasound images using fuzzy logic on coefficient of variation. Biomed. Signal Process. Control 23, 93-103 (2016) doi:10.1016/j.bspc.2015.08.001

13. N. Damodaran, S. Ramamurthy, S. Velusamy, G.K. Manickam, Speckle noise reduction in ultrasound biomedical b-scan images using discrete topological derivative, Ultrasound Med. Biol. 38 (2) (2012) 276-286.

14. M. Tsakalakis, N.G. Bourbakis, Ultrasound image despeckling /denoising based on a novel multi-transducer architecture, in: 2014 International Conference on Bioinformatics and Bioengineering, 10-12 Nov., Boca Raton, 2014, pp. 62-68.

15. L. Jun, C. Guangmeng, H. Bo, Image denoising based on fuzzy in wavelet domain, in: IMTC 2005 Instrumentation and Measurement Technology Conference, Ottawa, Canada, 2005, pp. 2019-2023, 16-19May.

\section{AUTHORS PROFILE}

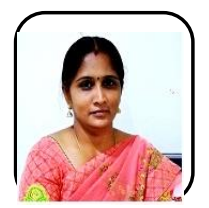

Nageswari $\mathbf{P}$ was born in Namakkal, Tamilnadu. She received bachelors and masters degree in mathematics from Periyar University, Tamilnadu, India, 2004 and 2006 respectively. She has been awarded Master of Philosophy in mathematics by Periyar University, Salem, Tamilnadu. Currently she is a Research scholar in the department of mathematics, Erode Arts and Science College. Her research interests include ultrasound image processing and fuzzy set theory.

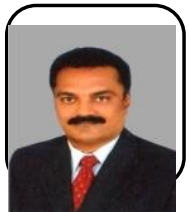

applications

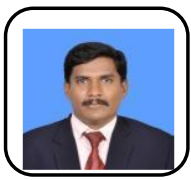
University, Chennai. He has held the position of Associate Professor and Researcher within the Centre for Electronics and Communication Engineering, Mahendra Engineering College, Tamilnadu, India. His main research interests are in the areas of image processing, artificial intelligent techniques. Erode Arts and Science College, Erode, Tamilnadu, India, MSc in 1984 from Madras University and MPhil in 1995 areas of interest are computer algebra systems and equations differentia

Manivel K was born in Namakkal, Tamilnadu. He received Master degree in Applied Electronics from Anna University of Technology Coimbatore, Tamilnadu, India, 2010. He has been awarded Ph.D., degree for his Research work in the area of image processing by Anna

Rajan $\mathbf{S}$ is an Associate Professor of Mathematics at

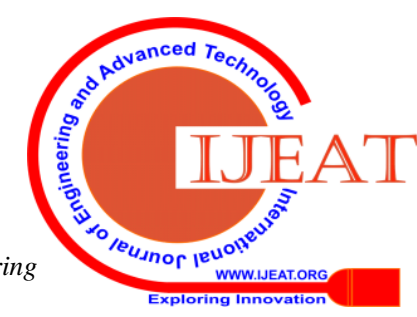

